\title{
La Pintada: Un sitio patrimonial en riesgo de desaparición
}

Ernesto Rivas Árevalo

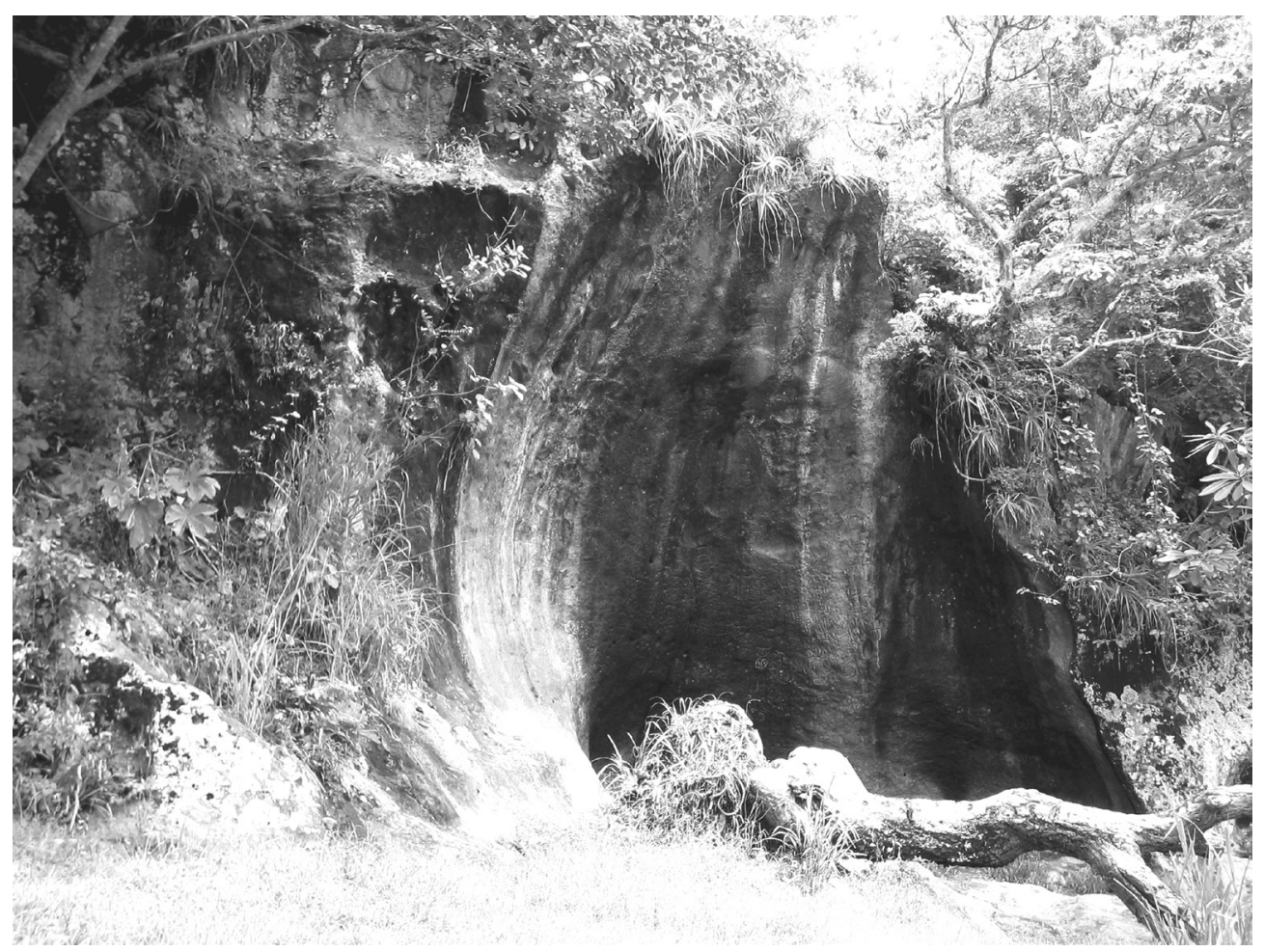

La Pintada está situada a la orilla del río Titihuapa, departamento de San Vicente, a unos 70 kilómetros de San Salvador, y muy vecina al municipio de San Isidro, departamento de Cabañas. La cueva es bastante conocida por los lugareños y su nombre ha sido mencionado por diversos investigadores, entre los que cabe destacar: Atilio Peccorini (1913), Rodolfo Barón Castro (1942), Wolfgang Haberland (1954) y Elisenda Coladán (1998). Al lugar se le conoce con varios nombres, entre otros, La Pintada, la Cueva Pintada o la Piedra Pin- 


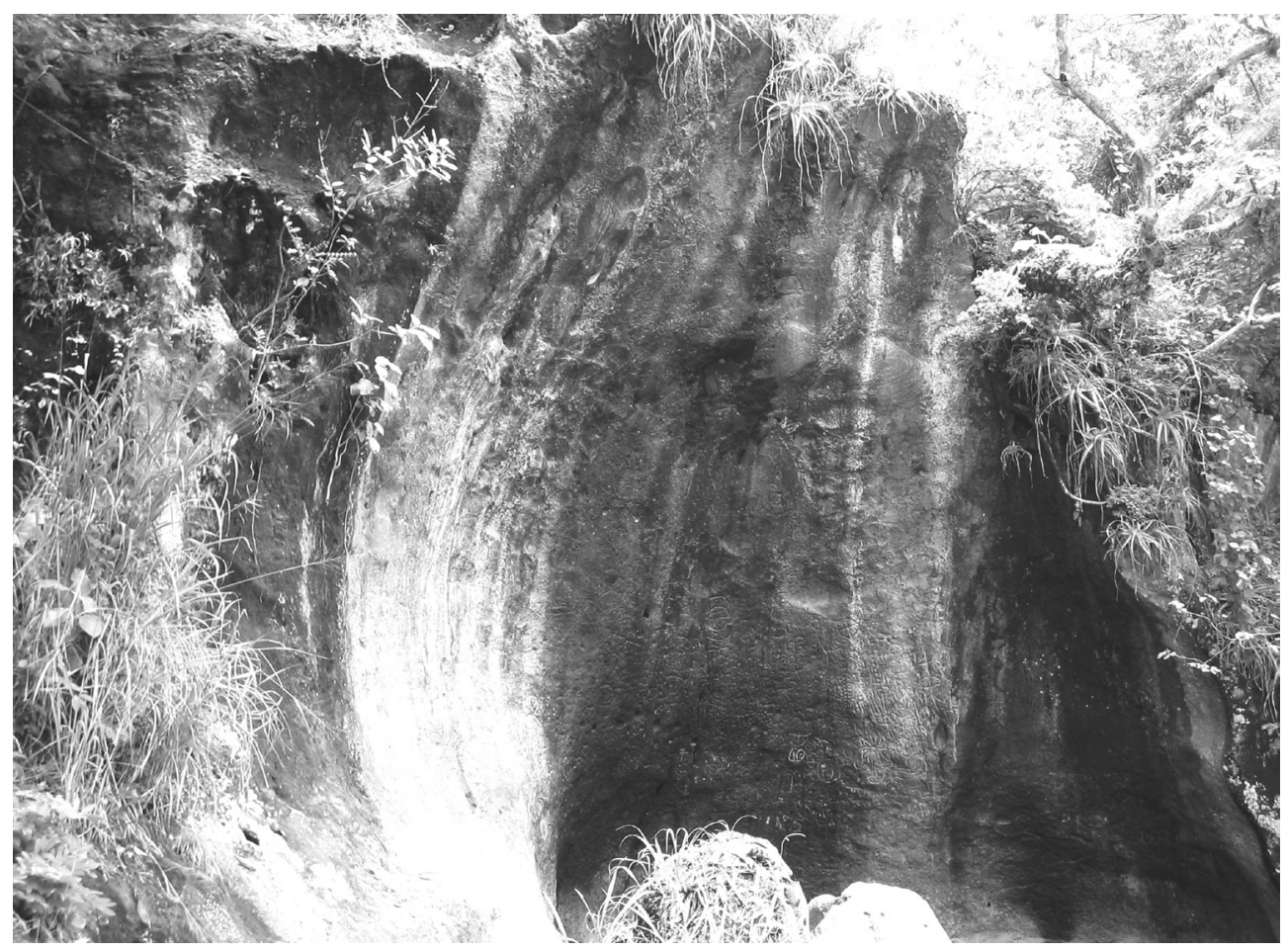

tada. Pero, en realidad se trata de un paredón al descubierto de forma cóncava. En la superficie hay grabados que tienden a la abstracción pero en los que también se pueden distinguir formas de seres humanos, animales y figuras geométricas. La Pintada está a la orilla del río, lugar donde hasta el momento, se descarta la posibilidad de que haya habido algún asentamiento.

Logicamente nos hacemos diversas preguntas. ¿Cuál fue la intencionalidad de la creación de estos petrograbados? ¿Cuál era la funcionalidad y el significado de estas figuras? A lo mejor eran símbolos mágico-religiosos ${ }^{1}$. ¿Lugar sagrado? Nos reconfortaría la idea de que probablemente se trataba de una interpretación hombre-naturaleza, puntos referenciales de caracter práctico (medio de comunicación) o puramente una expresión estética. Tales sugerencias sobre el significado de los signos son, sin duda, especulativas ya que, partiendo de nuestro contexto cultural y temporal, difícilmente lograremos dar una interpretar acertada del pensamiento del hombre precolombino.

\footnotetext{
1 "Las cuevas en los pueblos antiguos mesoamericanos eran lugares sacros, los mayas las consideraban como lugares de entrada al inframundo." Véase, Jarquín A, y Martínez E. "Problemática y resultado de las investigaciones arqueológicas en la zona de embalse de la Presa San Lorenzo, El Salvador" Ponencia presentada en el VI Congreso Centroamericano de Antropología, El Salvador, 2006.
} 
La cueva ya fue objeto de estudio anteriomente ${ }^{2}$ y se han encontrado similitudes con otros sitios rupestres dentro y fuera de El Salvador. ${ }^{3}$ Sin pretender desvirtuar los logros alcanzados hasta el momento, desafortunadamente muchos de estos estudios rupestres hechos son abordamientos descriptivos y no interpretativos. Esto ilustra la dificultad y complejidad del tema, así como la falta de nuevos estudios de profundización, que planteen nuevos enfoques. Todo eso es comprensible dado el caso que existen muchas limitaciones. $\mathrm{Mu}-$ chos de esos estudios dependen de un buen financiamiento y de la necesidad de llevarlos a cabo en un determinado lugar. Indudablemente, en El Salvador existen otros yacimientos rupestres que requieren también atención.

Otro aspecto importante es la metodología de trabajo por seguir. La complejidad de los signos requiere paradigmas de estudios que engloben ciertos razonamientos y la participación de otras disciplinas. En la búsqueda por descifrar la connotacion de los signos, se deben tener en cuenta referentes etnográficos, áreas geográficas, similitudes morfológicas, patrones repetitivos de símbolos, aspectos topográficos de la zona, aspectos lingüísticos, técnicas estilísticas utilzadas en el procedimiento de la elaboración de los petrograbados, etc., etc. Imprescindible es el valiosísimo aporte de disciplinas como la antropología, la arqueología, la linguiística u otras que den una interpretación partiendo del punto de vista de su propia área de estudio. Sin embargo, debemos tener en cuenta que muchas de las interpretaciones que se hagan siguen teniendo un carácter subjetivo y poco demostrable científicamente hablando.

Actualmente, en El Salvador se conoce la existencia de más de 60 sitios con presencia gráfico-rupestres (M. Escamilla.R., 2007). Diversos estudios nos indican que La Pintada debe situarse dentro de un contexto más amplio. Esta obra de petrograbados no se trata de un aspecto cultural aislado sino de un componente integral, parte de la red de los pueblos indígenas diseminados por toda Mesoamérica. Todavía no se ha profundizado con estudios interpretativos de las figuras de La Pintada, pero buena base la forman los estudios descriptivos ya existentes. Mayor claridad nos la darán estudios posteriores o

\footnotetext{
2 En el 2005 se llevó a cabo la denominada Misión Arqueológica Franco-Salvadoreña.

3 "La iconografía revela intrigantes semejanzas entre estos y más sitios de El Salvador y Honduras, ....". Ponencia para el II. Congreso Centroamericano de Arqueología (San Salvador, 23-26 de octubre de 2007), P.Costa y S. Perrot-Minnot.
} 


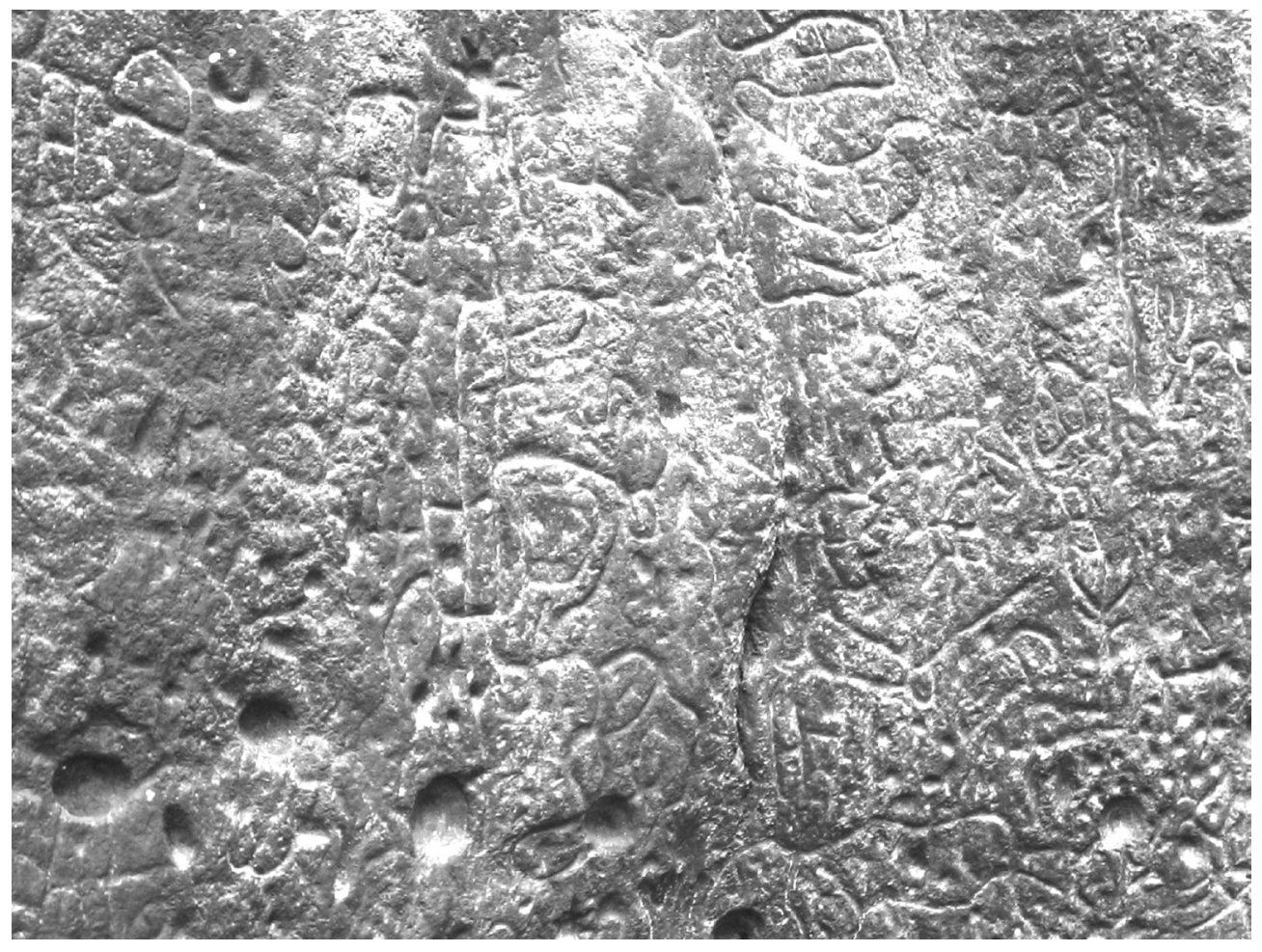

estudios comparativos hechos en otros $\operatorname{sitios}^{4}$ que muestren rasgos comunes, así como diferencias respecto a su simbología. Esto servirá como referencia para dar una interpretación más detallada.

El fechamiento de los petrograbados de La Pintada todavía no se ha determinado. Debemos tener en cuenta que, por toda Mesoamérica, existen lugares similares a La Pintada; yacimientos que cronológicamente corresponden a un mismo período ${ }^{5}$, lo mismo que centros ceremoniales de gran importancia pertenecientes a ese mismo período. A simple vista, La Pintada no podrá tener

\footnotetext{
4 En la gruta del Espíritu Santo, situada en la villa de Corinto (San Francisco Gotera, departamento de Morazán), también se pueden encontrar petrograbados con representaciones antropomorfas y figuras zoomorfas. Sin embargo, según Elisenda Coladán estas representaciones muestran cierta originalidad respecto a otras encontradas en El Salvador y Centroamérica. Véase: Elisenda Coladán, Las pinturas rupestres del oriente de El Salvador, versión digital, 1998. Veáse también El Diario de Hoy, artículo: Petrograbados indígenas, 11 de marzo 2005.

5 P. Costa y S. Perrot-Minnot dicen que, de acuerdo a las semejanzas estilísticas entre la Pintada de Titihuapa y la Cueva de los Fierros sugieren un posible fechamiento Clásico Tardío (600-900 d.C). A ese mismo período pertenecen centros ceremoniales como Chichen Itza, Uxmal, Xunantunich, etc., esto sólo para mencionar algunos lugares de renombre.
} 
la grandeza de otros lugares, pero es una de las tantas muestras de obras precolombinas $^{6}$ de El Salvador, y un tema único de ser estudiado.

\section{Vulnerabilidad del lugar y la necesidad de un plan de emergencia}

Como herederos de este legado cultural de nuestros antepasados, es obligación de todo salvadoreño, hacer suyo el compromiso de proteger estas manifestaciones artísticas, conservarlas y difundirlas a generaciones futuras.

Actualmente, La Pintada se encuentra en total abandono, su estado de conservación es deplorable. Tal pareciera que el lugar no ha recibido la atención y la valorización que se merece. Tanto el gobierno del municipio más cercano, como otras instancias gubernamentales, de quienes depende mucho su preservación, no han adoptado las medidas convenientes que garanticen su protección, como por ejemplo, declararla Zona Protegida. En el pasado, ha habido intentos simbólicos de hacer algo al respecto y hasta se han hecho recomendaciones a organismos locales de parte de arqueólogos ${ }^{7}$.

Cabe señalar que este abandono de la Pintada no es una excepción. Lo mismo puede verse en otros lugares como las ruinas de Quelepa situadas en la hacienda El Obraje en San Miguel, lugar donde sistemáticamente se ha dado el saqueo. ${ }^{8}$ Existen otros ejemplos semejantes a este. La Pintada se encuentra a la intemperie y las influencias climáticas como la lluvia y la erosión dañan la superficie. Sin embargo, un mayor peligro lo forman los daños ocasionados por la gente. Ese lugar es visto como lugar de esparcimiento y recreación. A menudo se puede encontrar toda clase de basura, huellas de fogatas encendidas al pie del paredón, etc.; escenas reconocibles de cualquier botadero de basura. El destrozo no termina allí porque, por su fácil acceso y por estar desprovisto de protección, se pueden encontrar grafitis y rayones sobre la piedra.

\footnotetext{
6 Debemos tener en cuenta de que si nombramos a tales obras rupestres: manifestaciones artísticas; estaremos aceptando y afirmando que se trata de Arte rupestre. Comparto el criterio de Patricio Bustamante Días cuando dice: "Todas aquellas obras que presumiblemente fueron concebidas y elaboradas con el fin de transmitir o almacenar conocimiento, no pueden ser clasificadas como objetos puramente ligados a la estética." No cabe duda que se pudo tratar de una obra con algotra destinación.

7 Los investigadores Eric Gelliot, Philippe Costa y Nadège Ramel y el estudiante Ismael Crespín, dieron a la Casa de la Cultura de San Isidro Cabañas unos rótulos para colocarlos con el fin de evitar depredaciones de parte de la la gente para que ésta respetase por lo menos un poco fragilidad de los petrograbados.

8 La Prensa Gráfica, 10 de julio del 2005.
} 
Sin duda toda toda alteración de los trazos de los motivos rupestres dificultará aún más cualquier estudio en el futuro.

Es necesario rescatar esa herencia del pasado, es necesario realizar un plan de rescate; un plan de emergencia que termine con el deterioro progresivo de $\mathrm{La}$ Pintada y que prevenga su destrucción posterior. Todo esto también con miras a evitar delitos patrimoniales al lugar. Es necesario trabajar en su restauración y recuperación.

\section{Referentes bibliográficos}

Bustamante, Días Patricio, ¿Arte?Rupestre, Análisis de la eficacia de un concepto actualmente en uso, En Rupestreweb, htt://rupestreweb.tripod.com/obrasrupestres.html

Coladán, Elisenda, Las pinturas rupestres del oriente de El Salvador. En XI simposio de Investigaciones Arqueológicas en Guatemala, 1997 (editado por J.P Laporte y H. Escobedo). pp. 660-671. Museo Nacional de Arqueolgía y Etnología, Guatemala. (versión digital).

Costa P. y S. Perrot-Minnot, Ponencia para el II. Congreso Centroamericano de Arqueología (San Salvador, 23-26 de octubre de 2007).

Escamilla, Marlon. Las montañas del norte y sus manifestaciones gráfico rupestres. Temporada 2007 Proyecto Arte Rupestre de El Salvador. Departamento de Arqueología. CONCULTURA, El Salvador, 2007.

-El Salvador rupestre: Reconocimiento y registro de sitios. En XX simposio de Investigaciones Arqueológicas en Guatemala, 2006 (editado por J.P. Laporte, B. Arroyo y H. Mejía), pp. 1219-1233. Museo Nacional de Arqueología y Etnología, Guatemala. (Versión digital).

Jarquín A, y Martínez E. "Problemática y resultado de las investigaciones arqueológicas en la zona de embalse de la Presa San Lorenzo, El Salvador" Ponencia presentada en el VI Congreso Centroamericano de Antropología, El Salvador, 2006.

Peccorini, Atilio. Algunos datos sobre arqueología de la República del Salvador. Conferencia pronunciada en el Salón de la Sociedad Americanistas en la sesión del 7 de Diciembre de 1912. 


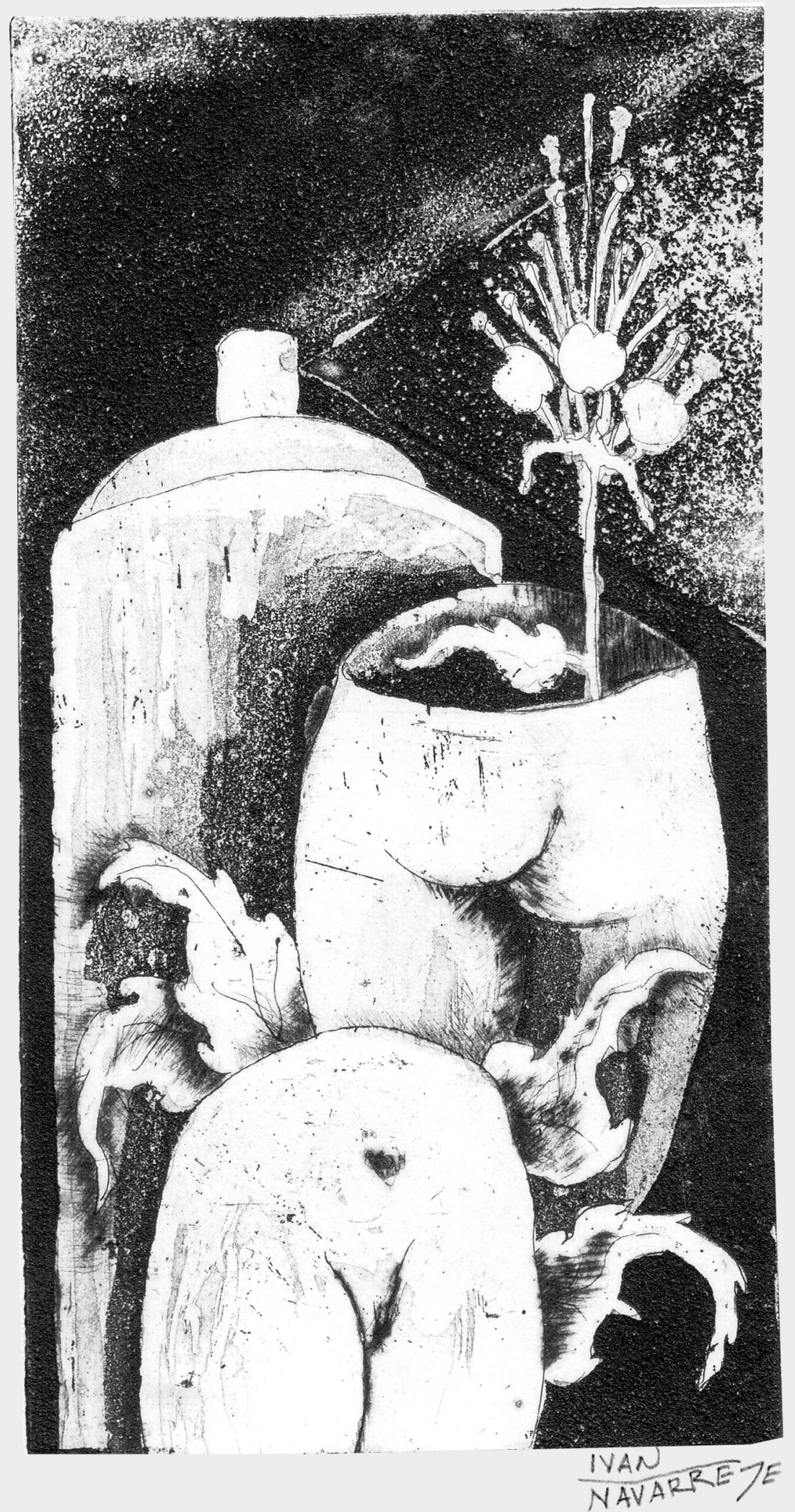




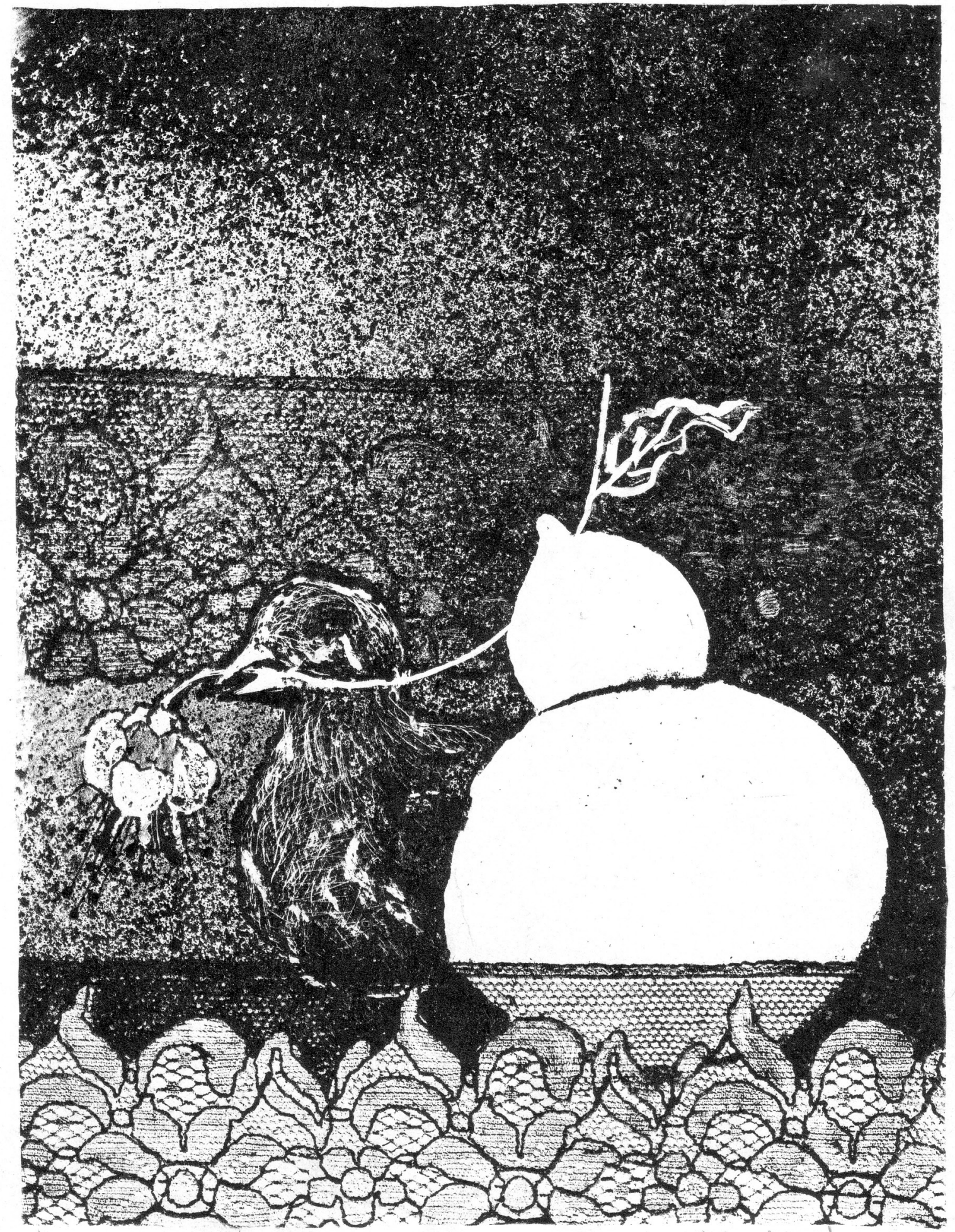

$\frac{\text { IVAN }}{\text { NAUARQEZE }}$ 